\title{
Efeito de Variáveis Climáticas no Crescimento Mensal de Pinus taeda e Araucaria angustifolia em Fase Juvenil
}

\author{
Sebastião do Amaral Machado ${ }^{1}$, Naiara Teodoro Zamin ${ }^{1}$, \\ Rodrigo Geroni Mendes Nascimento ${ }^{1}$, Angelo Alberto Pacheco dos Santos ${ }^{1}$ \\ ${ }^{1}$ Departamento de Ciências Florestais, Universidade Federal do Paraná - UFPR, Curitiba/PR, Brasil
}

\begin{abstract}
RESUMO
O objetivo deste trabalho foi construir curvas de produção e de incremento corrente mensal (ICM), e analisar o efeito de variáveis climáticas no crescimento mensal em diâmetro e altura de Pinus taeda L. e Araucaria angustifolia (Bert.) O. Kuntze, no período de três anos, em fase juvenil. Para tanto, foram selecionadas 30 árvores de cada espécie, sendo, mensalmente, entre junho de 2006 e julho de 2009, medidas as variáveis diâmetro e altura. As variáveis climáticas são provenientes de duas estações meteorológicas do SIMEPAR, próximas aos plantios monitorados. Para a análise do crescimento, foram desenvolvidas curvas de incremento e produção mensais, bem como análise da matriz de correlação dos dados e regressão pelo processo stepwise. $\mathrm{Na}$ análise do crescimento, observaram-se maiores ICMs entre outubro e fevereiro. As matrizes de correlação indicaram forte relação do ICM com temperatura, pressão atmosférica e fotoperíodo. Os modelos ajustados por stepwise, incluindo variáveis climáticas, apresentaram boas estatísticas de ajuste.
\end{abstract}

Palavras-chave: curva de produção, incremento corrente mensal, modelagem do crescimento, matriz de correlação.

\section{Effect of Climate Variables on the Monthly Growth of Pinus taeda and Araucaria angustifolia at Young Age}

\begin{abstract}
The objective of this research was to construct curves of yield and current monthly increment (CMI) and analyze the effect of climate variables on the current monthly increment of diameter and height of young Pinus taeda L. and Araucaria angustifolia (Bert.) O. Kuntze during a threeyear period. To this end, 30 trees of each species were selected and had their diameter and height measures taken monthly between June 2006 and July 2009. The climatic variables were obtained from two meteorological stations of SIMEPAR, located near the monitored stands. For growth assessment, curves of current monthly increment and yield were developed. Analysis of correlation matrixes among the studied variables and regression by stepwise process were also carried out. In the growth analysis, higher CMIs were observed between October and February. The correlation matrixes indicated that CMI correlated strongly with temperature, atmospheric pressure and photoperiod. The models adjusted by the stepwise process, including climate variables, showed good fit statistics.
\end{abstract}

Keywords: yield curve, current monthly increment, growth modeling, correlation matrix. 


\section{INTRODUÇÃO}

O crescimento das árvores consiste no acréscimo de camadas celulares na raiz, no tronco e nos galhos, modificando o tamanho e a forma das plantas. Esse crescimento ocorre de acordo com a Lei dos Rendimentos Decrescentes, com a curva de produção em forma sigmoidal. Essa curva se caracteriza pelo crescimento, primeiramente, acelerado, até atingir o ponto de inflexão, quando passa a crescer a menores taxas, até atingir estabilização no crescimento, identificada como valor máximo da produção biológica ou valor assintótico (Assmann, 1970).

Vários estudos têm sido realizados sobre esse comportamento (Turnbull, 1963; Assmann, 1970; Machado, 1978; Prodan et al., 1997; Scolforo, 1998), comprovando a veracidade da formação de curvas sigmoidais ao longo da vida das árvores. Porém, pouco se sabe a respeito da curva de produção dentro de um ano vegetativo, considerando o mês como unidade de tempo. Pressupõe-se apenas que o ritmo de crescimento decresce no inverno e possui forma acelerada na primavera e no verão, podendo apresentar similaridade com o comportamento do crescimento biológico ao longo de toda a vida do indivíduo.

Assmann (1970) correlacionou o crescimento da árvore dentro de um ano vegetativo com a curva em função da idade, concluindo que, assim como na curva gerada ano a ano, o ritmo de crescimento no decorrer de um ano (mês a mês) apresenta taxas crescentes de incremento até atingir um ponto máximo, seguido de decréscimos, caracterizando uma curva unimodal. Esse autor também verificou que as taxas de incremento no decorrer de um ano não estão relacionadas apenas com as condições climáticas, mas também com as reservas energéticas que a planta acumulou no último período de crescimento.

Segundo Scolforo (1998), conhecer o crescimento e a produção, presente e futura, de árvores e povoamentos florestais é fundamental para viabilizar o planejamento da atividade florestal; assim, neste contexto, a modelagem biológica se torna uma ferramenta útil na determinação do crescimento e da produção. Além disso, esse autor citou a necessidade de se obterem relações cada vez mais eficientes na representação da realidade, o que tem motivado inúmeras tentativas de modelagem dos fatores ambientais, como climáticos e edáficos, em associação com os fatores biológicos de crescimento e produção.

Modelos baseados em processos constituemse em relações cientificamente embasadas, que necessariamente contenham uma relação de causaefeito, sejam estas físicas ou biológicas (Stape et al., 2004). Maestri (2003) e Temps (2005) afirmaram que modelos baseados no clima sugerem que a mudança global climática pode influenciar o crescimento das árvores; portanto, torna-se necessário o desenvolvimento de modelos de crescimento e produção que incluam variáveis climáticas, que possam responder a essas mudanças.

Tendo em vista a crescente demanda na determinação de modelos de crescimento que incluam fatores ambientais como variáveis independentes, aliada ao fato de pouco se saber sobre o crescimento mensal dos indivíduos, estudos voltados para esse foco da modelagem florestal são necessários, uma vez que pesquisas desta natureza em nível de um ano vegetativo e respectivos incrementos correntes mensais (ICM) são raras e mesmo inéditas, principalmente para espécies de elevado valor comercial e científico, como é o caso de Pinus taeda L. e Araucaria angustifolia (Bert.) O. Kuntze.

Desta forma, o objetivo deste trabalho foi construir curvas de produção e de incremento corrente mensal (ICM), e analisar o efeito das variáveis climáticas no crescimento mensal em diâmetro e altura das espécies Pinus taeda e Araucaria angustifolia, no período de três anos, bem como verificar a contribuição dessas variáveis na composição de modelos de crescimento e produção.

\section{MATERIAL E MÉTODOS}

\section{1. Área de estudo}

Os dados desta pesquisa são provenientes de um plantio de Araucaria angustifolia localizado em Campina Grande do Sul e outro de Pinus taeda presente no município de Curitiba, ambos no Estado do Paraná. 
O município de Campina Grande do Sul encontra-se localizado no primeiro planalto paranaense, a uma altitude de 918 metros sobre o nível do mar (s.n.m.), inserido na Região Metropolitana de Curitiba a aproximadamente $27 \mathrm{~km}$ da capital paranaense. O clima predominante da região é Cfb, segundo a classificação de Köppen, também denominado clima temperado subtropical, com temperaturas amenas e agradáveis ao longo do ano. De acordo com estações meteorológicas do SIMEPAR, no período de junho de 2006 a julho de 2009, a precipitação média anual do local foi de $1.286 \mathrm{~mm}$, com médias de temperatura máxima de $22,68{ }^{\circ} \mathrm{C}$ e mínima de $12,57^{\circ} \mathrm{C}$. As coordenadas geográficas do plantio de Araucaria angustifolia alvo deste estudo são $25^{\circ} 19,073^{\prime} \mathrm{S}$ e $49^{\circ} 07,123^{\prime} \mathrm{W}$.

A capital paranaense, Curitiba, está localizada a $90 \mathrm{~km}$ do Oceano Atlântico, com uma altitude de 934 m s.n.m, no primeiro planalto paranaense. Apresenta clima Cfb de acordo com a classificação de Köppen, com verões amenos e chuvas bem distribuídas ao longo do ano. A precipitação média anual na região de Curitiba para os três anos deste estudo foi de $1.251 \mathrm{~mm}$, com média de temperatura máxima de $23,52{ }^{\circ} \mathrm{C}$ e mínima de $13,83{ }^{\circ} \mathrm{C}$, de acordo com estações meteorológicas do Sistema de Meteorologia do Paraná (SIMEPAR). O plantio de Pinus taeda estudado encontra-se sobre as coordenadas geográficas $25^{\circ} 14,568^{\prime} \mathrm{S}$ e $49^{\circ} 0,756^{\prime} \mathrm{W}$.

A estação meteorológica de Pinhais dista em média $10 \mathrm{~km}$ do plantio de A. angustifolia; já a estação de Curitiba está localizada a menos de 500 metros do plantio de P. taeda, no Campus III da Universidade Federal do Paraná.

\subsection{Coleta de dados}

Foram selecionadas para realização deste estudo 30 árvores de Araucaria angustifolia, com espaçamento $4 \times 4 \mathrm{~m}$, e 30 árvores de Pinus taeda com espaçamento $3 \times 3 \mathrm{~m}$. Estes povoamentos foram analisados mensalmente nos períodos de 1,2 e 3 anos de idade para a primeira espécie, e 2, 3 e 4 anos para a segunda.

A seleção das árvores ocorreu de forma aleatória, sempre analisando o estado fitossanitário dos indivíduos. Árvores bifurcadas, tortuosas ou com algum outro defeito foram excluídas da seleção.
Todas as árvores selecionadas receberam uma plaqueta metálica numerada, permitindo, desta maneira, um melhor controle e individualização das árvores em análise.

No início de cada mês, no período de junho de 2006 a julho de 2009, mediram-se o diâmetro a $10 \mathrm{~cm}$ do solo $\left(\mathrm{D}_{10 \mathrm{~cm}}\right)$ no plantio de Araucaria angustifolia, e o diâmetro a 1,3 m do solo (DAP) no plantio de Pinus taeda. Para tanto, utilizou-se o paquímetro até os diâmetros atingirem $6,0 \mathrm{~cm}$ e, posteriormente, a fita métrica graduada em milímetros. Na utilização do paquímetro, eram feitas duas medidas ortogonais, das quais se extraiu a média aritmética. O local de medição foi marcado com um gabarito e tinta spray, com o objetivo de todas as medidas serem realizadas no mesmo local. Para a mensuração da variável altura, utilizou-se régua altimétrica com precisão de $1,0 \mathrm{~cm}, \mathrm{em}$ ambos os plantios analisados.

\subsection{Análise do período de crescimento}

Para a análise do crescimento e da produção mensais, foram desenvolvidas curvas de produção, ano a ano, no período de três anos vegetativos, a fim de comparar a variação no comportamento do crescimento a cada ano e a cada mês de medição. Foram também construídos gráficos de incrementos correntes mensais médios (ICMs) dos plantios, buscando-se interpretar os períodos de maior e menor crescimento das espécies no decorrer de um ano, e correlacionar essas variações com as variáveis meteorológicas.

\subsection{Variáveis climáticas}

Foram obtidas bases de dados meteorológicos mensais provenientes das estações meteorológicas de Pinhais e de Curitiba, as quais foram selecionadas pela proximidade com os plantios, sendo essas bases de dados cedidas pelo SIMEPAR. Dentre as informações analisadas, estão: precipitação $(\mathrm{mm})$, temperaturas mínima, média e máxima $\left({ }^{\circ} \mathrm{C}\right)$, pressão atmosférica $(\mathrm{hPa})$, radiação média incidente na superfície $\left(\mathrm{W} / \mathrm{m}^{2}\right)$ e umidade relativa (\%). Além disso, calculou-se o fotoperíodo mensal médio (h/ dia) para a latitude de cada plantio (Equação 1), conforme segue: 


$$
D=\frac{2\left\{\arccos [-\operatorname{tg}(\varphi) * \operatorname{tg}(\delta)]+0,83^{\circ}\right\}}{15}
$$

em que: $\mathrm{D}=$ fotoperíodo (horas); $\phi=$ latitude do lugar; $\delta=$ declinação do sol.

A partir disso, foram elaboradas matrizes de correlação entre o incremento corrente mensal (ICM) em altura e diâmetro frente às variáveis climáticas, a fim de identificar quais são as mais relacionadas com o crescimento de Araucaria angustifolia e de Pinus taeda. Para as variáveis que apresentaram maior correlação, foram elaborados gráficos que representam essa relação, considerando os meses do ano no eixo ' $\mathrm{x}$ ' e as variáveis climáticas e dendrométricas no eixo ' $y$ '.

Para complementar a análise do efeito das variáveis climáticas sobre o crescimento mensal em diâmetro e altura para essas duas espécies, foram ajustados modelos de produção via regressão passo a passo ou stepwise.

Para o ajuste dessas equações de produção, foram utilizadas como variáveis dependentes a produção mensal em diâmetro e em altura, e como variáveis independentes o tempo (mês e ano) e as variáveis climáticas, assim como suas transformações nas formas logarítmica, inversa e quadrática.

A seleção do modelo mais eficiente para cada variável nos três períodos vegetativos e para cada espécie foi baseada no coeficiente de determinação ajustado e corrigido $\left(\mathrm{R}_{\mathrm{aj}}^{2}\right)$, no erro padrão $\mathrm{da}$ estimativa em percentagem $\left(\mathrm{S}_{\mathrm{yx} \%}\right)$ e com base no valor de F.

Para o ajuste dos modelos, foram feitos testes para determinar o mês inicial da curva de produção ao longo de um ano vegetativo para cada espécie, em que se avaliou a forma e o padrão da curva de produção com a alteração do mês de início do crescimento. Desta forma, tomou-se como mês inicial (mês zero) o mês de julho para A. angustifolia e o mês de junho para $P$. taeda. Após definido o mês de início, foram atribuídos valores aos meses, variando de 0 a 12, sendo essas as variáveis independentes no ajuste dos modelos de produção mensal. Segundo Brown (1974a), esse período é conhecido como estação ou época de crescimento. A estação de crescimento é um exemplo de ciclos de eventos, ou seja, eventos que se repetem em certos intervalos de tempo mais ou menos contínuos.

\section{RESULTADOS E DISCUSSÃO}

\subsection{Crescimento e produção mensal de Araucaria angustifolia}

Pode-se perceber que as curvas de produção em diâmetro de Araucaria angustifolia (Figura 1) não apresentaram forma sigmoidal característica, exceto no terceiro ano, em que há tendência ao valor assintótico. Isto pode ser explicado pela grande variação do incremento corrente mensal ao longo do ano, o qual não apresentou a forma unimodal comumente exibida pelas curvas de incremento (Assmann, 1970; Prodan et al., 1997).

Verificou-se uma tendência de crescente produção ao longo dos anos de análise (Figura 1). Isto reflete, no primeiro ano, a adaptação da espécie ao sítio e, nos anos seguintes, crescente produção seguindo a tendência do crescimento biológico, em que os indivíduos, quando jovens, crescem a elevadas taxas.

A variável diâmetro apresentou produção média anual de 1,97 cm para os três anos de estudo; além disso, as curvas apresentaram-se pouco sinuosas, tendendo a uma reta no primeiro ano, seguida de crescente sinuosidade nos anos subsequentes (Figura 1). Para altura, o crescimento acumulado médio foi de $1,09 \mathrm{~m}$ ao ano.

Nos gráficos de incremento corrente mensal (ICM) de A. angustifolia, foram observados picos no crescimento de diâmetro para os três anos de estudo; contudo, o máximo ICM variou no período de outubro a fevereiro. Para altura, o ICM máximo observado se deu em abril, seguido de um período de baixas taxas de crescimento influenciadas pela dormência da gema apical nos meses de junho a outubro.

O início do período vegetativo da variável altura se deu em agosto, fato possivelmente relacionado ao maior período de dormência das gemas apicais em comparação com o câmbio. Segundo Koehler (2009), o gênero Araucaria apresenta uma redução quase absoluta da atividade mitótica e do alongamento da gema apical no final do período vegetativo. Além disso, pode-se verificar a forma sigmoidal clássica das curvas de produção para essa variável. 

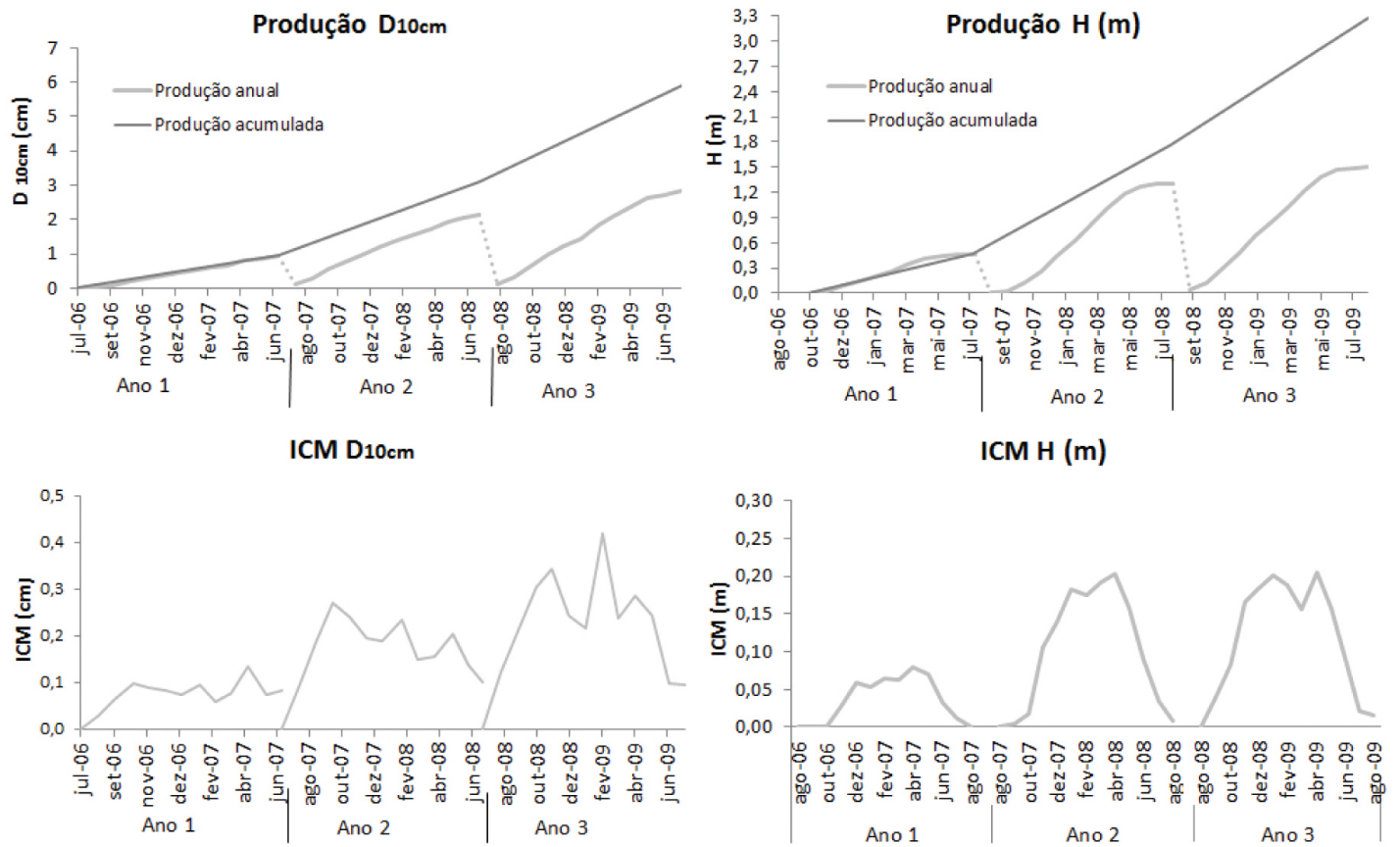

Figura 1. Curvas de produção e incremento corrente mensal (ICM) de diâmetro e de altura de Araucaria angustifolia.

Figure 1. Yield and current monthly increment (CMI) curves of diameter and height for Araucaria angustifolia.

As curvas de incremento corrente mensal para a variável altura, respeitadas as variações, apresentaram comportamento regular, visto que atingiram de maneira acelerada seu valor máximo, seguido de um decréscimo acelerado das taxas de crescimento. $\mathrm{O}$ incremento corrente mensal máximo para os anos 1, 2 e 3 analisados ocorreram no mês de abril, e foram de 0,080 m, 0,203 m e 0,206 m, para os três anos, respectivamente.

Zanon (2007), em estudo de crescimento mensal em diâmetro de Araucaria angustifolia, utilizando cintas dendrométricas, no período de três anos, observou que o início do período de crescimento ocorreu em setembro. As curvas de produção apresentaram padrão sigmoidal, com máximo incremento nos meses com maiores temperaturas e com redução na atividade cambial, entre os meses de março e abril. Essa autora também observou um incremento anual médio de $0,24 \mathrm{~cm} \cdot \mathrm{ano}^{-1}$ para indivíduos de povoamentos com idade de 40 e 60 anos, valor abaixo ao do povoamento jovem estudado no presente trabalho, que apresentou produção média de $1,97 \mathrm{~cm} \cdot \mathrm{ano}^{-1}$ para o período de 1 a 3 anos de idade.
Em estudo realizado por Figueiredo Filho et al. (2003), com espécies da Floresta Ombrófila Mista, foram realizadas medições no início de cada estação do ano por meio de cintas dendrométricas, num período de três anos. Esses autores observaram que os maiores incrementos em diâmetro de Araucaria angustifolia ocorreram no verão, seguido de primavera, outono e inverno. O incremento médio anual em diâmetro observado foi de $0,13 \mathrm{~cm} \cdot \mathrm{ano}^{-1}$, sendo inferior ao observado neste estudo, já que o incremento médio mensal para os três anos avaliados foi de $0,165 \mathrm{~cm} \cdot \mathrm{mês}^{-1}$ e o anual de $1,97 \mathrm{~cm} \cdot \mathrm{ano}^{-1}$. Esta diferença se explica pelo fato de os indivíduos deste estudo serem pertencentes a um povoamento jovem (1 a 3 anos) e os indivíduos analisados por Figueiredo Filho et al. (2003) estarem presentes em uma floresta multiânea, incluindo indivíduos de diferentes classes diamétricas e sujeitos a elevados níveis de competição.

Hess et al. (2009), analisando a variação do crescimento em diâmetro de Araucaria angustifolia para três regiões do Rio Grande do Sul, obtiveram um incremento médio anual de $0,98 \mathrm{~cm} \cdot \mathrm{ano}^{-1}$. Conforme já evidenciado anteriormente, o 
incremento anual observado na presente pesquisa foi superior, o que pode ser explicado pela idade dos indivíduos estudados, visto que esses autores avaliaram indivíduos de 20 a 33 anos de idade.

\subsection{Crescimento e produção mensal de Pinus taeda}

As curvas de produção de Pinus taeda para o período de um ano vegetativo, considerando como unidade de tempo o mês (Figura 2), apresentaram padrão sigmoidal. Para a altura, o valor assintótico é atingido no mês de maio, devido ao período de dormência das gemas apicais. $\mathrm{O}$ mesmo não ocorre para o diâmetro, o qual reduz suas taxas de incremento nos meses de inverno, mas sem que haja paralisação total do crescimento.

Machado et al. (2010) verificaram por meio de análise de Cluster que o crescimento mensal em altura para Pinus taeda, no período de abril a julho, apresenta padrão diferenciado em comparação aos demais períodos do ano, sendo classificado como um grupo à parte pela análise multivariada realizada. Segundo esses autores, essa classificação ocorre devido às características de baixo crescimento e quase repouso vegetativo ou dormência das gemas apicais, nos meses mais frios do ano.

Para o diâmetro, foi observado que o segundo ano de avaliação apresentou maior produção anual $(5,03 \mathrm{~cm})$ quando comparada ao ano $1, \operatorname{com} 4,74 \mathrm{~cm}$, e ao ano 3, com 4,29 cm. A variável altura apresentou produção semelhante para os três anos avaliados, com média de $1,92 \mathrm{~m}$, atingindo valor assintótico entre os meses de maio e junho (Figura 2).

$\mathrm{O}$ incremento corrente anual em diâmetro não sofreu variações para os três anos estudados, mantendo uma taxa semelhante de crescimento, caracterizando uma produção acumulada com tendência linear, conforme pode ser observado na Figura 2. Contudo, pode-se observar uma redução na produção em diâmetro no terceiro período de análise, o que pode estar relacionado às menores temperaturas registradas neste ano de medição, conforme pode ser observado na Figura 3.

Nas curvas de incremento de Pinus taeda (Figura 2), observou-se que a variável diâmetro, assim como para Araucaria angustifolia, apresentou picos de crescimento no decorrer dos anos de avaliação. Além disso, o terceiro período vegetativo apresentou um comportamento atípico, com
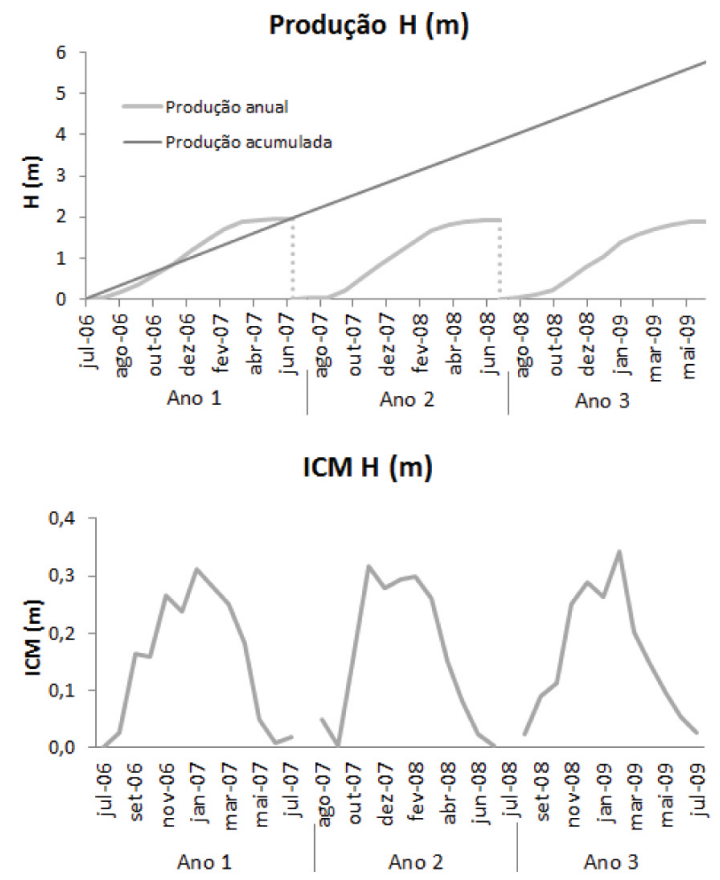

Figura 2. Curvas de produção e incremento corrente mensal (ICM) para diâmetro e altura de Pinus taeda. Figure 2. Yield and current monthly increment (CMI) curves of diameter and height for Pinus taeda. 
(a)

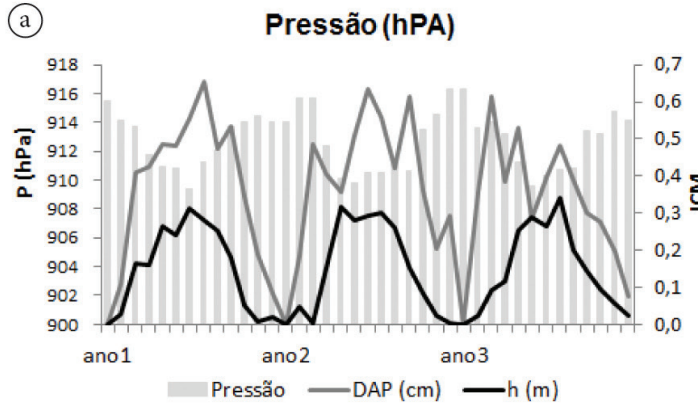

Fotoperíodo (h/dia)

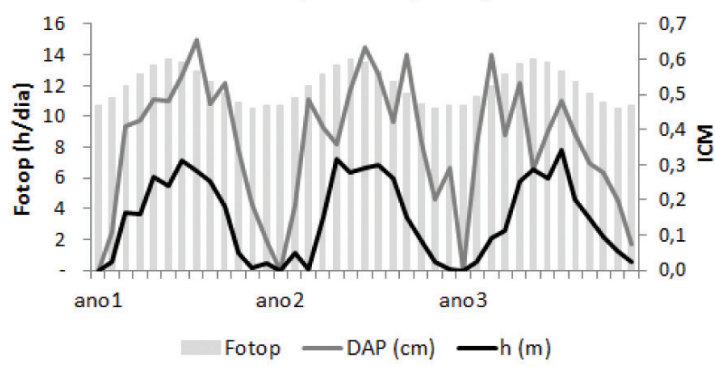

Temperatura mínima $\left({ }^{\circ} \mathrm{C}\right)$

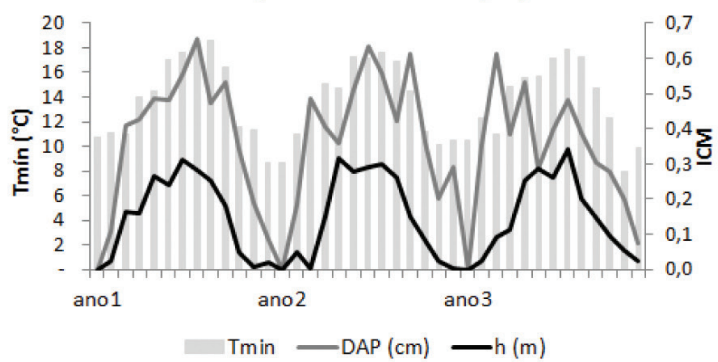

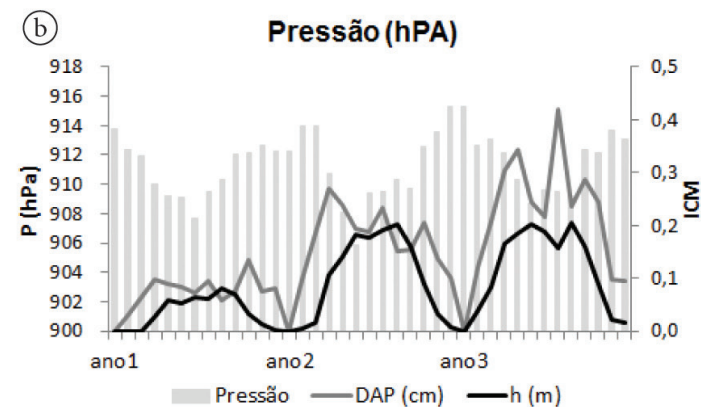

Fotoperíodo (h/dia)

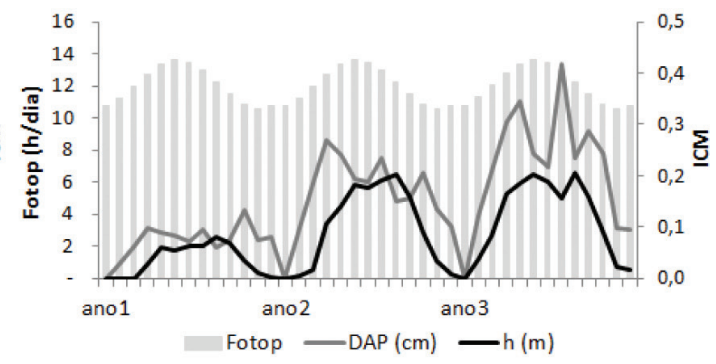

Temperatura mínima $\left({ }^{\circ} \mathrm{C}\right)$

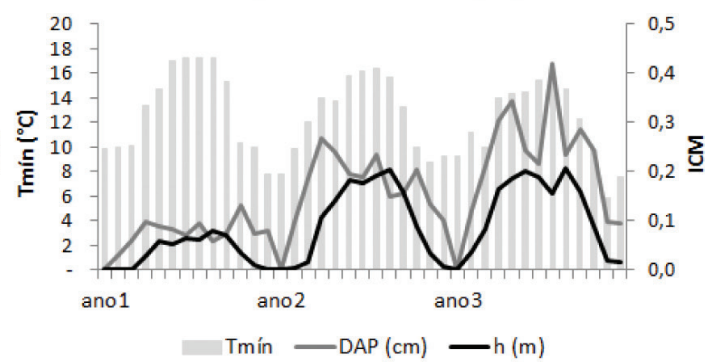

Figura 3. Correlação entre incrementos correntes mensais (ICM) em diâmetro e altura com variáveis climáticas mais correlacionadas: a) Pinus taeda e b) Araucaria angustifolia.

Figure 3. Correlation among current monthly increment (ICM) in diameter and height with climatic variables more correlated: a) Pinus taeda and b) Araucaria angustifolia.

máximo incremento em diâmetro no mês de setembro $(0,61 \mathrm{~cm})$, ou seja, logo no início do período, apresentando picos posteriores, contudo com taxas inferiores de crescimento. Para os anos 1 e 2, o máximo ICM ocorreu em fevereiro $(0,65 \mathrm{~cm})$ e janeiro $(0,63 \mathrm{~cm})$, respectivamente, estando dentro dos padrões esperados, visto que normalmente os maiores incrementos ocorrem no verão.

As curvas de incremento corrente mensal para a variável altura, respeitadas as variações, apresentaram comportamento esperado, ascendendo até um valor máximo, com taxas de crescimento decrescentes a partir deste ponto máximo. Os incrementos correntes mensais máximos para os três períodos analisados ocorreram na época mais quente do ano, nos meses de janeiro $(0,31 \mathrm{~m})$, novembro $(0,32 \mathrm{~m})$ e fevereiro $(0,34 \mathrm{~m})$, respectivamente, para os anos 1,2 e 3 .

Em estudo realizado por Serpe \& Watzlawick (2008), encontrou-se um incremento diamétrico anual, em plantios com quatro anos de idade, espaçamento $2,5 \times 2,0 \mathrm{~m}$, de aproximadamente $2,50 \mathrm{~cm}$, diferindo dos valores obtidos neste estudo, em que se detectou $4,69 \mathrm{~cm} \cdot \mathrm{ano}^{-1}$, considerando a mesma idade. Contudo, vale ressaltar que o presente estudo possui um maior espaçamento $(3,0 \times 3,0 \mathrm{~m})$, o que pode ser o motivo do crescimento superior em diâmetro. 


\subsection{Variáveis climáticas}

Por meio da análise da matriz de correlação, podem-se perceber baixas correlações do ICM em diâmetro de $A$. angustifolia com as variáveis climáticas, ao passo que a altura apresentou correlação de moderada a forte com algumas dessas variáveis, conforme apresentado na Tabela 1. Esse resultado mostrou que o incremento diamétrico dessa espécie é pouco sensível às variações climáticas, sendo, de certa forma, mais influenciado pelas reservas energéticas e atividades metabólicas intrínsecas das árvores, o que está relacionado a uma fase de adaptação ao sítio de A. angustifolia. Desta forma, nessa fase de desenvolvimento, o crescimento está sendo conduzido pelo ritmo endógeno de crescimento inerente a cada espécie (Luz, 2011), uma vez que as variações climáticas não seriam suficientemente drásticas e regulares de maneira a preponderar e a induzir alterações na atividade cambial (Oliveira, 2007).

Para Pinus taeda, perceberam-se maiores valores de coeficiente de correlação de Pearson entre as variáveis de crescimento e as variáveis climáticas analisadas, quando comparada com a mesma análise feita para A. angustifolia.

Segundo Brown (1974a), os diferentes padrões de crescimento das árvores estão relacionados com as condições sazonais a que as plantas estão expostas, uma vez que os indivíduos adaptam seu crescimento para suportar o estresse sazonal ao qual estão submetidos. Assmann (1970) também afirmou que as taxas de crescimento ao longo do ano flutuam de acordo com as condições do clima. Entretanto, ressaltou que o ritmo de crescimento não depende exclusivamente das condições climáticas do ano de avaliação, mas também das condições ocorrentes em anos anteriores, especialmente em meses em que os brotos apicais são formados.

Observou-se, ainda, na Tabela 1, correlação inversa dos incrementos de diâmetro e altura em relação à pressão atmosférica em ambas as espécies. Considerando-se que a pressão atmosférica varia ao longo do ano de forma contrária às demais variáveis climáticas, sendo mais elevada no inverno e mais baixa no verão, podem-se entender os índices de correlação inversa observados, que chegam até -0,9.

Ferreira \& Couto (1981) avaliaram a pressão atmosférica como uma variável fortemente correlacionada com a altitude, sendo esta um fator de grande influência sobre o desenvolvimento do crescimento em altura de povoamentos de Eucalyptus spp localizados em Minas Gerais e Espírito Santo. Em todas as espécies estudadas por esses autores, o aumento da altitude acarretou um menor crescimento em altura.

Ainda segundo Schober (1949) citado por Assmann (1970), plantios localizados em maiores altitudes tendem a iniciar seu crescimento mais tardiamente no ano vegetativo e apresentar maior duração do período de crescimento ao longo do

Tabela 1. Matriz de correlação entre variáveis climáticas e o ICM de Araucaria angustifolia e de Pinus taeda, nos três anos de avaliação.

Table 1. Correlation matrix among climatic variables and the CMI of Araucaria angustifolia and Pinus taeda, in the three years of evaluation.

\begin{tabular}{ccccc} 
Variável climática & \multicolumn{2}{c}{ Araucaria angustifolia } & \multicolumn{2}{c}{ Pinus taeda } \\
\cline { 2 - 5 } & ICM-D $_{\mathbf{1 0} \mathrm{cm}}$ & ICM-H & ICM-DAP & ICM-H \\
\hline Pp & 0,15 & 0,18 & 0,32 & 0,36 \\
P & $-0,34$ & $-0,65$ & $-0,63$ & $-0,91$ \\
Tmáx & 0,16 & 0,47 & 0,60 & 0,76 \\
Tmed & 0,26 & 0,58 & 0,69 & 0,84 \\
\hline Tmín & 0,35 & 0,65 & 0,74 & 0,88 \\
\hline UR & 0,44 & 0,49 & 0,54 & 0,45 \\
\hline Rad & 0,20 & 0,09 & 0,38 & 0,58 \\
\hline F & 0,45 & 0,64 & 0,69 & 0,89 \\
\hline
\end{tabular}

ICM- $\mathrm{D}_{10 \mathrm{~cm}}$ : incremento corrente mensal do diâmetro medido a $10 \mathrm{~cm}$ de altura; ICM-DAP: incremento corrente mensal do diâmetro a altura do peito; ICM-H: incremento corrente mensal da altura total; Pp: precipitação; P: pressão atmosférica; Tmáx: temperatura máxima; Tmed: temperatura média; Tmín: temperatura mínima; UR: umidade relativa; Rad: radiação solar; F: fotoperíodo. 
ano, tendo sido observado por esse autor que o crescimento em diâmetro iniciou cerca de duas a quatro semanas depois na altitude de $500 \mathrm{~m}$, comparativamente à de $300 \mathrm{~m}$. Com isso, percebe-se a grande influência que a altitude e, consequentemente, a pressão atmosférica exercem sobre o crescimento em diâmetro e altura das árvores, conforme expresso na matriz de correlação.

As variáveis climáticas fotoperíodo e temperaturas máxima, média e mínima apresentaram maior correlação com as taxas de crescimento da espécie $P$. taeda. Apesar de mais baixa, esta correlação também é observada para $A$. angustifolia. Segundo Brown (1974b), a luz é um dos constituintes do ambiente que apresentam maior influência sobre o desenvolvimento das árvores, uma vez que o crescimento e a forma da árvore são diretamente afetados pelas condições de intensidade, qualidade e duração da luz. Esse autor afirmou também que a temperatura é um fator importante no crescimento, porque influencia em muitas atividades fisiológicas que afetam o metabolismo das plantas. Ainda, segundo Lüttge \& Hertel (2009), os estímulos ambientais que afetam a atividade cíclica de crescimento anual são principalmente fotoperíodo e temperatura.

A independência do crescimento em diâmetro de A. angustifolia em relação às variáveis climáticas pode estar relacionada ao fato de o câmbio estar protegido por uma grossa camada de casca, o que reduz os efeitos do clima sobre sua atividade de divisão celular (Machado et al., 2010); o mesmo não ocorre para a altura, visto que os meristemas apicais estão mais expostos às variações climáticas, as quais afetam até mesmo seu período de dormência durante o ano.

Por meio da análise gráfica (Figura 3), pode-se observar que o aumento da produção de Araucaria angustifolia a cada ano, nos três anos de estudo, não sofreu influência das variáveis climáticas, comprovando a ideia de que a produção crescente ao longo dos anos ocorre devido à idade juvenil dos indivíduos que estão com total vitalidade e em crescente produção até que o ponto de inflexão da curva de produção ano a ano seja atingido. $\mathrm{Na}$ análise gráfica de ambas as espécies, percebe-se que o incremento de altura e diâmetro cresce com o aumento da temperatura e do fotoperíodo, fator que evidencia de maneira geral o fato de as árvores crescerem menos no inverno do que no verão.

Puchalski et al. (2006) verificaram que a temperatura média, o número de geadas, a umidade relativa e o fotoperíodo são variáveis climáticas importantes para ocorrência natural de A. angustifolia. Observou-se também que algumas destas variáveis também foram importantes para o estudo do crescimento mensal em altura e diâmetro dessa espécie, corroborando, portanto, com os resultados obtidos nesta pesquisa.

Souza (2004) realizou estudo sobre crescimento de ipê-felpudo em relação às variáveis climáticas em Linhares, Espírito Santo, concluindo que a temperatura não afetou muito o crescimento dessa espécie. Segundo esse autor, esse fato foi atribuído à pouca variação dessa variável climática ao longo do ano na região do estudo, uma vez que quanto mais próxima aos trópicos, a sazonalidade é determinada pela variação pluviométrica. No entanto, na região da presente pesquisa, há grande variação da temperatura no decorrer das estações do ano, razão pela qual essa variável afeta bastante o crescimento durante $o$ ano vegetativo.

Bognola (2007) observou que a precipitação, a temperatura mínima e a temperatura máxima são as variáveis climáticas que mais afetam o incremento de Pinus taeda, diferindo deste estudo, uma vez que foi encontrada baixa correlação entre o incremento e a precipitação. Pode-se observar (Figura 4) que os níveis de precipitação são bastante variáveis ao longo do ano. Apesar de haver variação da precipitação ao longo do ano não há períodos de déficit hídrico na região desta pesquisa, conforme a classificação de Koppen em clima Cfb; por isso, a baixa correlação dessa variável com o crescimento.

\subsection{Modelagem da produção mensal para Araucaria angustifolia}

Neste item, é apresentado o ajuste das equações de produção, por meio do método stepwise, possuindo como variáveis dependentes o diâmetro e a altura, e como variáveis independentes as unidades de tempo (mês de medição e idade) e as variáveis climáticas.

$\mathrm{O}$ melhor ajuste pelo processo stepwise para estimar a produção em diâmetro, a partir de medições 

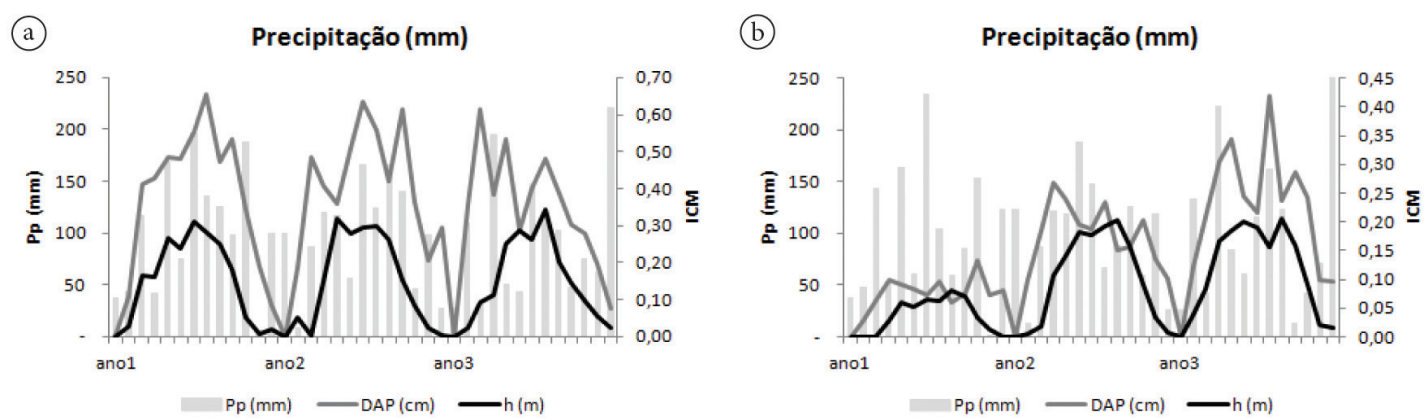

Figura 4. Correlação entre incrementos correntes mensais (ICMs) em diâmetro e altura com a precipitação: a) Pinus taeda e b) Araucaria angustifolia.

Figure 4. Correlation among current monthly increment (ICM) in diameter and height with precipitation: a) Pinus taeda and b) Araucaria angustifolia.

mensais para a espécie A. angustifolia, apresentou como variáveis independentes do modelo: a idade do povoamento, o mês de medição (variando de $0 \mathrm{a}$ 12) e a raiz quadrada da radiação. Segundo Ferreira (2009), a radiação solar possui também importante efeito sobre o crescimento, uma vez que a fotossíntese é iniciada pela radiação fotossinteticamente ativa.

Diversamente, para a estimativa da produção mensal em altura, foram selecionadas as variáveis: idade do povoamento, o mês de medição e o inverso da precipitação. As equações estão apresentadas na Tabela 2 com seus respectivos coeficientes e variáveis.

As equações ajustadas para A. angustifolia apresentaram bons indicativos de ajuste e precisão, com elevados coeficientes de determinação $\left(\mathrm{R}_{\mathrm{aj}}^{2}\right)$, porém os erros padrões da estimativa em percentagem $\left(\mathrm{S}_{\mathrm{yx} \%}\right)$ foram relativamente altos, e valores de $\mathrm{F}$ significativo a $1 \%$ de probabilidade, evidenciando a eficácia da determinação da produção mensal em função das variáveis inseridas no modelo.

\subsection{Modelagem da produção mensal para} Pinus taeda

Na definição de modelos que estimem a produção mensal de diâmetro e altura em função das variáveis meteorológicas para Pinus taeda (Tabela 3), pode-se observar que a regressão stepwise que apresentou o melhor ajuste para o diâmetro incluiu as variáveis independentes mês de medição e exponencial da precipitação na equação de melhor ajuste.
Pode-se verificar que a precipitação foi selecionada novamente na modelagem stepwise, apesar da baixa correlação $(0,32)$ com o incremento diamétrico, decorrente da ausência de déficit hídrico durante todo o ano.

$\mathrm{Na}$ estimativa da produtividade de povoamentos monoclonais de híbridos de Eucalyptus grandis e E. urophylla, Stape et al. (1997) concluíram que a produtividade é muito dependente da precipitação ocorrida ao longo do processo de desenvolvimento das plantas. Souza (2004) ainda ressaltou que uma grande variação da precipitação proporciona diversos problemas nas plantas, uma vez que a água tem grande importância para o desenvolvimento dos vegetais.

Para a altura, foram selecionadas as variáveis mês de medição e exponencial da temperatura mínima, lembrando que essa variável climática apresentou elevado coeficiente de correlação linear, conforme apresentado na matriz de correlação (Tabela 1). A seleção dessas variáveis climáticas, aliada aos bons resultados das estatísticas de ajuste e precisão (Tabela 3), confirma o efeito destas no crescimento mensal em diâmetro e altura de $P$. taeda.

Temps (2005) utilizou o procedimento da eliminação das variáveis 'backward' a fim de ajustar modelos de incremento corrente anual de altura dominante de Pinus taeda. Desta maneira, esse autor verificou que a precipitação média foi a variável que mais explicou a variabilidade da variável dependente, apresentando um coeficiente de determinação entre 0,51 e 0,53 . Além disso, esse autor fez análise gráfica 
Tabela 2. Estatísticas de ajuste e precisão da equação do crescimento acumulado em diâmetro a $10 \mathrm{~cm}$ e em altura total de Araucaria angustifolia, em função das variáveis selecionadas na modelagem.

Table 2. Statistics of adjustment and precision of the yield equations for diameter at $10 \mathrm{~cm}$ and for total height of Araucaria angustifolia as a function of the selected variables in the modelling.

\begin{tabular}{cccc} 
Modelo & $\mathbf{R}^{\mathbf{2}_{\mathbf{a j}}}$ & $\mathbf{S}_{\mathbf{y x} \%}$ & $\mathbf{F}$ \\
\hline$D=-1,5607+0,4334 I+0,1630 M+0,0391 \sqrt{ } \mathbf{R}$ & 0,906 & 26,64 & $112,48^{* *}$ \\
$H=-0,6691+0,2843 I+0,1128 M-0,5005(1 /(P p))$ & 0,869 & 35,09 & $77,75^{* *}$ \\
\hline
\end{tabular}

$\mathrm{I}=$ idade do povoamento; $\mathrm{M}$ = mês de medição; $\mathrm{R}=$ radiação; $\mathrm{Pp}=$ precipitação; "* significativo a $1 \%$ de probabilidade.

Tabela 3. Estatísticas de ajuste e precisão da equação do crescimento acumulado em diâmetro a 1,30 m e altura total de Pinus taeda em função das variáveis selecionadas na modelagem.

Table 3. Statistics of adjustment and precision of yield equations for diameter at $1,30 \mathrm{~m}$ and total height of Pinus taeda as a function of the selected variables in the modelling.

\begin{tabular}{cccc} 
Modelo & $\mathbf{R}^{\mathbf{2}}$ & $\mathbf{S}_{\mathbf{y} \mathbf{0}}$ & $\mathbf{F}$ \\
\hline$D=-0,1085+0,4369 M-1,08 \times 10^{-96} e^{\mathrm{Pp}}$ & 0,9856 & 8,11 & $1230,67^{* *}$ \\
$H=-0,1658+0,1910 M+3,50 \times 10^{-9} e^{\text {Tmin }}$ & 0,9690 & 13,22 & $562,62^{* *}$ \\
\hline
\end{tabular}

$\mathrm{M}=$ mês de medição; $\mathrm{Pp}=$ precipitação; Tmín= temperatura mínima; ${ }^{* *}=$ significativo a $1 \%$ de probabilidade.

com o objetivo de confirmar a relação observada na regressão, obtendo comportamento semelhante para ambas as técnicas de análise. Temps (2005) ainda observou que, com a inclusão da variável climática no modelo de crescimento e produção, a equação ajustada apresentou um ganho significativo na prognose da altura dominante, propiciando a redução do erro de estimativa em 7,5\%.

\section{CONCLUSÕES}

A curva de produção durante o período vegetativo, para ambas as espécies, nos três anos de estudo, apresentou a forma típica sigmoidal, similar à curva de produção ao longo de toda a vida da árvore.

A variável diâmetro para ambas as espécies sofreu menor influência das variáveis climáticas do que a altura.

O ICM em diâmetro e altura apresentou relação diretamente proporcional com o fotoperíodo e a temperatura, e relação inversa com a pressão atmosférica, para as duas espécies estudadas.

Por meio da matriz de correlação, análise gráfica e regressão stepwise, foi possível verificar que as variáveis climáticas apresentam efeito no crescimento mensal em diâmetro e altura para ambas as espécies estudadas, viabilizando seu uso na estimativa do crescimento e da produção mensal dessas variáveis.

\section{STATUS DA SUBMISSÃO}

Recebido: 10 set., 2012

Aceito: 27 jan., 2014

Publicado: 30 jun., 2014

\section{AUTOR(ES) PARA CORRESPONDÊNCIA}

\section{Sebastião do Amaral Machado}

Departamento de Ciências Florestais, Universidade Federal do Paraná - UFPR, CEP 80210-170, Curitiba, PR, Brasil e-mail: samachado@ufpr.br

\section{REFERÊNCIAS}

Assmann E. The principles of forest yield study - Studies in the organic production, structure, increment and yield of forest stands. Oxford: Pergamon Press; 1970.

Bognola IA. Unidades de manejo para Pinus taeda L. no planalto norte catarinense, com base em características do meio físico [tese]. Curitiba: Universidade Federal do Paraná; 2007.

Brown, CL. Secondary Growth. In: Zimmermann $\mathrm{MH}$, Brown CL, editor. Trees Structure and Function. Nova York: Spring-Verlag; 1974a.

Brown CL. Growth and Form. In: Zimmermann $\mathrm{MH}$, Brown CL, editor. Trees Structure and Function. Nova York: Spring-Verlag; $1974 b$.

Ferreira CA, Couto HTZ. A influência de variáveis ambientais no crescimento de espécies/procedências de 
Eucalyptus spp. nos estados de Minas Gerais e Espírito Santo. Boletim da Pesquisa Florestal 1981; (3): 9-35.

Ferreira MZ. Modelagem da influência de variáveis ambientais no crescimento e produção de Eucalyptus sp. [tese]. Lavras: Universidade Federal de Lavras; 2009.

Figueiredo Filho A, Hubie SR, Schaaf LB, Figueiredo DJ, Sanquetta CR. Análise do incremento em diâmetro com o uso de cintas dendrométricas em algumas espécies de uma Floresta Ombrófila Mista localizada no sul do Estado do Paraná. Revista Ciências Exatas e Naturais 2003; 5(1): 69-84.

Hess AF, Schneider PR, Finger CAG. Crescimento em diâmetro de Araucaria angustifolia (Bertol.) Kuntze em função da idade, em três regiões do Rio Grande do Sul. Ciência Florestal 2009; 19(1): 7-22.

Koehler AB. Modelagem biométrica e morfométrica em povoamentos jovens de Araucaria angustifolia (Bert.) KTZE., em Tijucas do Sul, Estado do Paraná. [tese] Curitiba: Universidade Federal do Paraná; 2009.

Lüttge U, Hertel B. Diurnal and annual rhythms in trees. Trees 2009; (23): 683-700. http://dx.doi.org/10.1007/ s00468-009-0324-1

Luz AL de OM. Análise da formação dos anéis de crescimento anual das árvores ao longo dum ciclo de actividade cambial [dissertação]. Lisboa: Instituto Superior de Agronomia, Universidade Técnica de Lisboa; 2011.

Maestri R. Modelo de crescimento e produção para povoamentos clonais de Eucalyptus grandis considerando variáveis ambientais [tese]. Curitiba: Universidade Federal do Paraná; 2003.

Machado SA. Studies in growth and yield estimation for Pinus taeda L. plantations in the State of Paraná [tese]. University of Washington; 1978.

Machado SA, Figura MA, Silva LCR, Nascimento RGM, Quirino SMS, Téo SJ. Dinâmica de crescimento de plantios jovens de Araucaria angustifolia e Pinus taeda. Pesquisa Florestal Brasileira 2010; 30(62): 165-170. http://dx.doi.org/10.4336/2010.pfb.30.62.165

Oliveira JM. Anéis de crescimento de Araucaria angustifolia (Bertol.) O. Kuntze: bases de dendroecologia em ecossistemas subtropicais montanos no Brasil [tese]. Porto Alegre: Universidade Federal do Rio Grande do Sul; 2007.
Prodan M, Peters R, Cox F, Real P. Mensura Forestal. San José: Deustsche Gesellschaf für Technische Zusammenarbeit; 1997.

Puchalski A, Mantovani M, Reis MS. Variação em populações naturais de Araucaria angustifolia (Bert.) O. Kuntze. associada a condições edafo-climáticas. Scientia forestalis 2006; 70: 137-148.

Serpe EL, Watzlawick LF. Avaliação de incremento diamétrico inicial em diferentes espécies de Pinus na região de Santa Maria do Oeste (PR). Revista Eletrônica lato Sensu - UNICENTRO 2008; (6). [cited 2010 ago. 1]. Available from: http://web03.unicentro. br/especializacao/Revista_Pos/P\%C3\%A1ginas/6\%20 Edi\%C3\%A7\%C3\%A3o/Agrarias/PDF/2-Ed6_CAAvali.pdf.

Souza CC. Modelo de crescimento, com variáveis ambientais, para o ipêfelpudo em diferentes espaçamentos [dissertação]. Piracicaba: Universidade de São Paulo; 2004.

Scolforo JR. Modelagem do crescimento e da produção de florestas plantadas e nativas. Lavras: UFLA/FAEPE; 1998.

Stape JL, Gomes NA, Assis TF. Estimativa da produtividade de povoamentos monoclonais de Eucaliptus grandis $\mathrm{x}$ urophylla no nordeste do Estado da Bahia - Brasil em função das variabilidades pluviométricas e edáficas. In: Anais do Iufro Conference on Silviculture and Improvement of Eucalyptus; 1997. Colombo: EMBRAPA/CNPF; 1997. p. 192-198.

Stape JL, Ryan MG, Binkley D. Testing the utility of the 3-PG model growth of Eucalyptus grandis $\mathrm{x}$ urophylla with natural and manipulated supplies of water and nutrients. Forest Ecology and Management 2004; 193: 219-234. http://dx.doi.org/10.1016/j.foreco.2004.01.031

Temps M. Adição da precipitação pluviométrica na modelagem do crescimento e da produção florestal em povoamentos não desbastados de Pinus taeda $L$. [dissertação]. Curitiba: Universidade Federal do Paraná; 2005.

Turnbull KJ. Population dynamics in mixed forest stands. A system of mathematical models of mixed stand growth and structure [tese]. University of Washington; 1963.

Zanon MLB. Crescimento da Araucaria angustifolia (Bertol.) Kuntze. diferenciado por dioicia [tese]. Santa Maria: Universidade Federal de Santa Maria; 2007. 